\title{
Constraints on the cosmic equation of state: Age conflict versus phantom energy
}

\section{Age-redshift relations in an accelerated universe}

\author{
J. Cepa ${ }^{1,2}$ \\ 1 Instituto de Astrofísica de Canarias, 38200 La Laguna, Tenerife, Spain \\ e-mail: jcn@ll.iac.es \\ 2 Departamento de Astrofísica, Facultad de Física, Universidad de la Laguna, 38071 La Laguna, Tenerife, Spain
}

Received 24 November 2003 / Accepted 24 March 2004

\begin{abstract}
The currently available values and confidence limits for $\Omega_{\mathrm{m} 0}, H_{0}$ and globular cluster ages still indicate that the dark energy that dominates the Universe could also be a form of quintessence or phantom energy. In fact, current cosmological values favor phantom energy. To increase the likelihood of a cosmological constant as dark energy instead of phantom energy, the possibilities seem to lie in reducing globular cluster ages, the Hubble constant, or both, and possibly advancing the epoch of globular cluster formation. For a set of possible dark energy equations of state that includes the cosmological constant, quintessence or phantom energy, age-redshift analytical expressions for null curvature universes that include ordinary matter are derived together with the corresponding ages for these universes. "Cosmic coincidences" are found for the currently accepted values of $\Omega_{\mathrm{m} 0}$ when evaluating the age of the Universe with a cosmological constant or a phantom energy as dark energies.
\end{abstract}

Key words. cosmology: theory - relativity - cosmology: cosmological parameters

\section{Introduction}

Curvature, age, the Hubble constant and energy density form a long-standing cosmological puzzle. Measurements of the cosmic microwave background radiation (CMBR) anisotropy have confirmed that the Universe is consistent with null, or nearly null, curvature (de Bernardis et al. 2000), as predicted by the inflation paradigm. However, a Euclidean universe composed of ordinary matter only has an age inconsistent with that derived from globular clusters (Bolte \& Hogan 1995). Moreover, galaxy cluster data (Carlberg et al. 1996) allow us to obtain only about $20-30 \%$ of the amount of matter required for achieving null curvature. This puzzle seemed to be solved by the discovery in 1998 of the first evidence for an accelerating universe based on distant type Ia Supernova (SNeIa) observations (Riess et al. 1998), which have been supported by additional data including more distant $\mathrm{SNe}$ (Riess et al. 2001). This acceleration implies the presence of a significant fraction of dark energy with an equation of state of negative pressure. The addition of the required amount of this energy to the observed amount of matter allows us to obtain the total amount of energy required for achieving null curvature. It also has the desired side effect of producing an older universe that can then be made consistent with independent age measurements.

However, the nature of this dark energy is still to be established. Moreover, its equation of state is still unknown. The first attempts rescued the cosmological constant as an ad hoc explanation, although their interpretation of the constant in terms of a vacuum energy is inconsistent by 124 orders of magnitude with respect to the required value, which leads us to assume that its value has to be zero for consistency. Other energy types, such as quintessence or phantom energy, have also been invoked, although their origin is no clearer than that of the cosmological constant.

Quintessences were introduced by Wetterich (1998), Caldwell et al. (1998), Ratra \& Peebles (1998) to avoid the extreme fine-tuning needed to allow a cosmological constant to be significant only at recent epochs. Quintessences are characterized by a scalar field whose evolution depends on its potential. The most popular potentials are inverse power and exponential laws (Kneller \& Strigari 2003 and references therein), although other possibilities have been considered (see, for example, Di Pietro \& Claeskens 2003). Both inverse power laws and exponential potentials converge to a unique solution for a broad range of different initial conditions.

Phanton energy (Caldwell 1999) violates the dominantenergy condition (Hawking \& Ellis 1973) that might allow the existence of wormholes. It also makes the Universe reach in a finite time a cosmic doomsday where all objects, from galaxies to nucleons, are ripped apart, a situation that has been termed the "big rip" (Caldwell 1999). Although some Chaplygin gas 
model generalization can avoid this big rip (González-Díaz 2003), the violation of the dominant energy condition and the difficulties in obtaining a stable phantom model (Carroll et al. 2003) renders this kind of energy more problematic than quintessences.

In the case of either quintessences, the cosmological constant or phantom energy, it seems established that the introduction of negative pressure energies is required to fit and harmonize the existing CMBR anisotropy, SNeIa, clusters of galaxies, large scale structure, Big Bang nucleosynthesis and age estimator data.

The incorporation of these energies changes the ageredshift relation and estimates of the age of the Universe. The aim of this article is to show the difficulty in avoiding phantom energies with the current age estimator data and to provide analytical age expressions for the homogeneous and isotropic case for the most likely equations of state for our Universe, considered constant, as restricted by the cosmological parameters commonly assumed. These include the cosmological constant, quintessence and phantom energy.

Thomas \& Kantowski (2000) provide an age-redshift relation using elliptical integrals for a universe composed of matter and a cosmological constant, but without providing an easy ready-to-use expression. In general, no analytical expressions of age as a function of redshift seems to be readily available in the literature for the currently most widely accepted cosmologies: flat universes of matter plus a cosmological constant, quintessence or phantom energy.

Although numerical integration allows us to obtain ages in a relatively straightforward way (see, for example, Hogg 2000), analytical solutions are faster to evaluate and have the advantage of providing more precise and ready results than numerical approaches. Moreover, analytical expressions provide the explicit dependence on energy densities, thereby easing their study. Finally, they constitute a reference for checking numerical solutions.

In Sect. 2 the basic equations are given. In Sect. 3 possible values for the equation of state, considered constant, are reviewed according to recent figures for the cosmological parameters, in Sect. 4 the general case of a non-constant equation of state is outlined, and in Sect. 5 analytical age-redshift relations and ages are obtained by solving the equations on a case by case basis for the range of the constant equations of state assumed.

\section{Basic equations}

The age-redshift relation can be obtained from the Friedmann equations. Using units defined by $c=G=1$, where $c$ is the speed of light and $G$ the constant of gravitation, the Friedmann equation representing energy balance can be written as

$H^{2}=\left(\frac{\dot{a}}{a}\right)^{2}=\frac{8 \pi}{3} \rho-\frac{\epsilon}{a^{2}}$,

where $a$ is the scale factor at a cosmological time $t, \rho$ the total energy density, $\epsilon$ the curvature parameter and the dot indicates the time derivatives.
The total energy density as a function of redshift, $z$, is defined to be

$\frac{8 \pi}{3} \rho=H_{0}^{2}\left(\Omega_{\mathrm{m} 0}(1+z)^{3}+\Omega_{\mathrm{w} 0}(1+z)^{3(1+w)}\right)$,

where subscript 0 indicate present-day magnitudes of timedependent functions, $\Omega_{\mathrm{m} 0}$ and $\Omega_{\mathrm{w} 0}$ are the present-day energy densities over the critical density of ordinary matter and of dark energy, respectively, $1+z=a_{0} / a$, and $w=p_{w} / \rho_{w}$ is the equation of state of dark energy given by the ratio of scalar pressure over energy density, which is assumed constant.

This assumption, a generalization of the cosmological constant that represents a barotropic fluid, lacks physical justification. A constant $w$ is valid for the cosmological constant only, since in general a time dependency is expected in quintessences and phantom energies. However, the absence of well motivated dark energy models based on fundamental physics and the difficulty to observe a time dependence of $w$ either by using the CMBR (Aurich \& Steiner 2003) or fits to luminosity distances (Di Pietro \& Claeskens 2003), makes this assumption a reasonable model-independent approximation to the equation of state of these energies (see Kneller \& Strigari 2003 and references therein for advantages and limitations of this approach). As a consequence, the constant $w$ values inferred represent effectives values weighted with respect to $\Omega_{\mathrm{w} 0}$ (Doran et al. 2001; Doran \& Lilley 2002). Nevertheless, a more general case is briefly outlined in Sect. 4.

For an $\epsilon=0$ universe, then, the age-redshift relation can be obtained using

$t=\frac{1}{H_{0}} \int_{z}^{\infty} \frac{\mathrm{d} z}{(1+z) \sqrt{\Omega_{\mathrm{m} 0}(1+z)^{3}+\Omega_{\mathrm{w} 0}(1+z)^{3(1+w)}}}$,

where $-1 / 3<w<-1$ for quintessences, $w=-1$ for the cosmological constant and $w<-1$ for phantom energies.

The age of the Universe, $t_{0}$, for a given combination of $\Omega_{\mathrm{m} 0}$, $\Omega_{\mathrm{w} 0}$ and the Hubble constant, $H_{0}$, can be obtained by setting the lower limit of (3) to $z=0$.

\section{Constraints on the cosmic equation of state}

Fits of observational data of good standard candles to distanceredshift relations can provide limits on the parameter $w$ of the equation of state. The main limitations of this method comes from the uncertainties in the extinction and evolution of the selected candles and in the curvature, $H_{0}$ and energy densities. Constraining the dark energy equation of state from the age of the Universe requires an accurate knowledge of $H_{0}, \Omega_{\mathrm{m} 0}$, the curvature and $t_{0}$, where $t_{0}$ is obtained from the age, $t_{\mathrm{GC}}$, of globular cluster or halo stars used as age indicators, plus the time, $t_{\mathrm{f}}$, elapsed from the Big Bang to globular cluster formation. For this article, the following parameters have been assumed:

1. A flat Universe $(\epsilon=0)$ as inferred from CMBR data. This result does not depend on the cosmological model (see, for example, Hu et al. 2001 ; Knox et al. 2001).

2. The Hubble constant $H_{0}=72 \pm 8 \mathrm{~km} \mathrm{~s}^{-1} \mathrm{kpc}^{-1}$ as obtained by the HST Key Project to Measure the Hubble Constant (Freedman et al. 2001), since it is derived from 
Table 1. Upper limits for $w$ obtained for the assumed values of $\Omega_{\mathrm{m} 0}$ and $t_{\mathrm{GC}}$ at $68 \%$ and $95 \%$ c.l. for globular cluster formation at $z=3$.

\begin{tabular}{ccccc}
\hline \hline$t_{0}(\mathrm{Gyr})$ & $\Omega_{\mathrm{m} 0}$ & $t_{\mathrm{GC}}(\mathrm{Gyr})$ & $w$ & $H_{0}\left(\mathrm{~km} \mathrm{~s}^{-1} \mathrm{kpc}^{-1}\right)$ \\
\hline 13.53 & 0.295 & 11.45 & -1.21 & 72 \\
11.98 & 0.295 & 10.00 & -0.56 & 72 \\
13.66 & 0.260 & 11.45 & -1.02 & 72 \\
12.06 & 0.260 & 10.00 & -0.52 & 72 \\
13.73 & 0.295 & 11.45 & -0.83 & 64 \\
11.88 & 0.295 & 10.00 & -0.25 & 64 \\
13.82 & 0.260 & 11.45 & -0.57 & 64 \\
11.92 & 0.260 & 10.00 & -0.23 & 64 \\
\hline
\end{tabular}

local Universe objects, whose distances are not measurably affected by the cosmological model assumed.

3. The density of matter can be obtained from the multiple source data statistical analysis of Turner (2002), which give $\Omega_{\mathrm{m} 0}=0.33 \pm 0.035$ (68\% c. 1 .).

4. The age of the Universe is obtained from the age of galactic globular clusters as $12.9 \pm 2.9 \mathrm{Gyr}$ at $95 \%$ c.l. using Hipparcos astrometric data (Carretta et al. 2000). From HST data the same authors estimate the epoch, $t_{\mathrm{f}}$, of formation of the galactic globular clusters to be $z=3$, which approximately corresponds to 2 Gyr depending on cosmology. This is the same $t_{\mathrm{f}}$ as assumed by Spergel et al. (2003). Hence, $10.0+t_{\mathrm{f}}$ Gyr represents an estimate for the minimum age of the Universe, that depends on the equation of state through the age-redshift relation.

From each of the $\Omega_{\mathrm{m} 0}$ lower limits derived from the $68 \%$ and $95 \%$ c.l. of this parameter, values of the equation of state, $w$, are obtained by numerical integration of (3) assuming null curvature and consistency with the lower limits for the ages of globular clusters derived from their $68 \%$ and $95 \%$ c.l. These values constitute upper limits for $w$ because smaller values would be consistent with a larger $t_{0}$ and/or larger $\Omega_{\mathrm{m} 0}$. Since $t_{\mathrm{f}}$ corresponds to $z=3$, an iterative procedure has been followed. Given that most of the uncertainties in the Hubble constant are systematic (Freedman et al. 2001), two $H_{0}$ values, corresponding to the mean and to the lowest boundary, have been considered. The latter again yields an upper limit for $w$ since lowering $H_{0}$ requires increasing $w$ for the same age and $\Omega_{\mathrm{m} 0}$. This procedure allows us to obtain the results of Table 1 for the upper limits of $w$ for the different combination of $68 \%$ and $95 \%$ c.l. of $\Omega_{\mathrm{m} 0}, t_{0}$ and $H_{0}$. Lower limits in $w$ have not been derived since more accurate age estimates are required because the age becomes less sensitive to $w$ when $w$ decreases (Fig. 1).

From the fit to distance-redshift relations using gravitational lenses and SN data, Waga \& Miceli (1999) derive $w<$ -0.7 (68\% c.1.) and $w<-0.6$ (95.4\% c.1.) for $\Omega_{\mathrm{m} 0}=0.3$. Perlmutter et al. (1999) obtained $w<-0.6$ (95\% c.l.) using SNeIa and large scale structure data. The age of the Universe was the procedure employed by Krauss \& Chaboyer (2003) from globular cluster age estimates to obtain $w<-0.7(68 \%$ c.1.) and $w<-0.45$ (95\% c.1.). A statistical analysis from WMAP (Wilkinson Microwave Anisotropy Probe) CMBR data combined with SNeIa and large scale structure data gives $w<-0.78$ at $95 \%$ c.l. (Spergel et al. 2003), with the previous $w>-1$. The results of Table 1 are then consistent with those obtained by other authors.

Although it is usually accepted that the most likely value that makes the existing data self-consistent is $w=-1$ (see, for example, Krauss \& Chaboyer 2003; Spergel et al. 2003), the fact is that quintessence $(-1 / 3>w>-1)$ cannot be ruled out on observational grounds using currently existing data. Moreover, the assumptions of this article do not allow us to rule out phantom energy $(w<-1)$. In fact, the mean values assumed for $\Omega_{\mathrm{m} 0}, t_{0}$, and $t_{\mathrm{f}}$ give $w=-4.95$ for $H_{0}=72 \mathrm{~km} \mathrm{~s}^{-1} \mathrm{kpc}^{-1}$ and $w=-1.49$ for $H_{0}=64 \mathrm{~km} \mathrm{~s}^{-1} \mathrm{kpc}^{-1}$. Taking into account that the $w$ values inferred are effective values (Sect. 2), then $w(t)$ must have been even smaller, which points strongly to an epoch of the Universe with $w<-1$.

An elementary analysis, varying only one of the parameters $\Omega_{\mathrm{m} 0}, t_{\mathrm{GC}}, t_{\mathrm{f}}$ and $H_{0}$ at a time, while keeping $w=-1$ fixed, gives the following results for the possible options to make the assumed mean values compatible with a cosmological constant instead of phantom energy:

1. Reducing $t_{\mathrm{f}}$. The age of the Universe derived using (3) and the assumed mean values for $\Omega_{\mathrm{m} 0}$ and $H_{0}$ is $12.7 \mathrm{Gyr}$, which gives no time for globular clusters to form. This possibility alone, then, is not enough.

2. Reducing $t_{\mathrm{GC}}$ to $10.7 \mathrm{Gyr}$, a possibility that is not excluded by the error bars of current age determinations.

3. Reducing $\Omega_{\mathrm{m} 0}$ to 0.18 , a value barely below the accepted lowest limit of 0.2 (Reichart et al. 1999). However del Popolo (2003) suggests that current $\Omega_{\mathrm{m} 0}$ values obtained from clusters of galaxies are underestimated by $20 \%$. In this case, not only does the possibility of reducing $\Omega_{\mathrm{m} 0}$ seem unlikely, but its value could even increase, thereby reducing even further the $t_{0}$ derived from (3) and making the need for phantom energy more likely for the current observational data to be consistent.

4. Reducing $H_{0}$ to $61 \mathrm{~km} \mathrm{~s}^{-1} \mathrm{kpc}^{-1}$, a value that seems to be excluded by the HST Key Project to Measure the Hubble Constant (Freedman et al. 2001), but that is consistent with other determinations using gravitational lenses (Fassnacht et al. 2002), the Sunyaev-Zel'dovich effect (Mauskopf et al. 2000) of SNeIa (Parodi et al. 2000), among others.

5. Increasing $\Omega_{\Lambda 0}$ to 1.12 to give $\Omega_{\text {total }}=1.45$, incompatible with current CMBR anisotropy data (e.g., Spergel et al. 2003; Hu et al. 2001).

Somewhat more rigorously, by parametric derivation of (3) under the integral sign, a Gaussian error distribution in $w$ centered on $w=-1$, with $w<-0.78$ (95\% c.l. of Spergel et al. 2003), gives $t_{0}=12.7 \pm 0.4 \mathrm{Gyr}$ at $95 \%$ c.l. for $\Omega_{\mathrm{m} 0}=0.33$ and $H_{0}=72 \mathrm{~km} \mathrm{~s}^{-1} \mathrm{kpc}^{-1}$. Taking $t_{\mathrm{GC}}$ at face value leaves at most only 0.2 Gyr for globular clusters to form or, equivalently, globular clusters should form around $z=17$ or earlier. Considering $\Omega_{\mathrm{m} 0}=0.26$ (95\% c.l. of Turner 2002, and within the values obtained by Spergel et al. 2003) gives $t_{0}=13.6 \pm 0.4 \mathrm{Gyr}$ at $95 \%$ c.l., which leaves $1.1 \mathrm{Gyr}$, or equivalently $z=5.3$ or earlier for globular clusters to form. This possibility could be consistent with the assumption that globular cluster formation can happen 


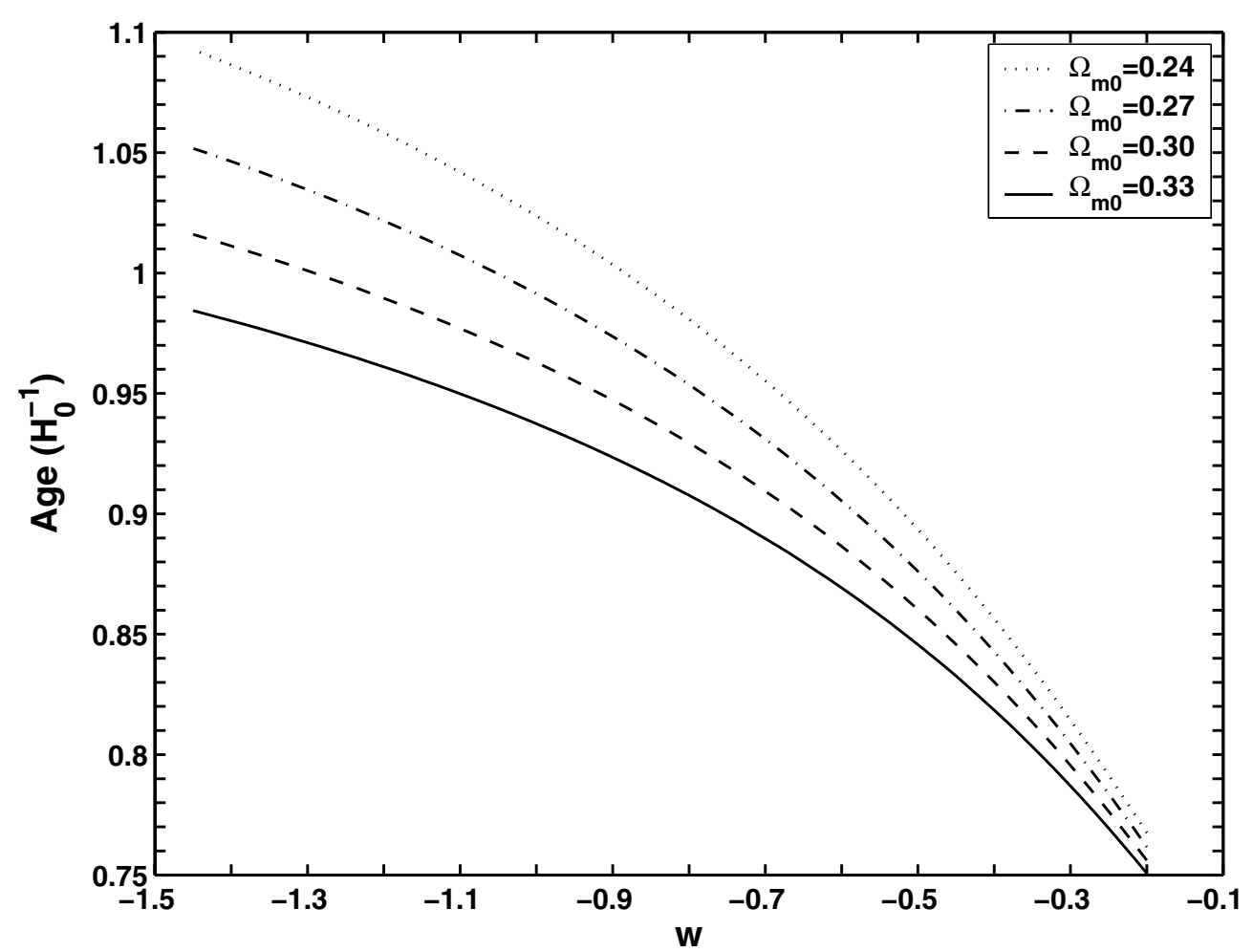

Fig. 1. Universe age versus the equation of state of dark energy, $w$, for a null curvature universe with ordinary matter. The age becomes less sensitive to $w$ as $w$ decreases.

as early as $z=6$ as claimed by some authors (van den Bergh 2001).

Although obtaining a conclusive result, then, requires more accurate data on $\Omega_{\mathrm{m} 0}, H_{0}, t_{\mathrm{GC}}$ and $t_{\mathrm{f}}$, there is prima facie qualitative evidence that the possibilities of reducing the current likelihood of phantom energy to favor a cosmological constant as dark energy reduces $t_{\mathrm{GC}}, H_{0}$ or both, and possibly advances the epoch of globular cluster formation.

\section{The time dependent equation of state}

\subsection{General case}

Except in the case of the cosmological constant, energy densities vary with redshift. While non-relativistic matter and radiation can be represented by constant $w$, this do not generally happen with quintessences and phantom energies, although in these cases a constant $w$ representing a weighted mean can be taken as an approximation.

As already stated in the introduction, quintessences were invoked to avoid the fine-tuning problem posed by the dominance of the cosmological constant in recent epochs only. Moreover, quintessences with $w(z)$ might present some additional advantages such as string theory compatibility by avoiding the existence of an event horizon (Cline 2001). Many quintessence models that differ in the form of the potential have been proposed (see Doran et al. 2001 for some potentials used).
Phantom energies have been represented by González Díaz (2003) by means of a suitable generalization of the Chaplygin gas model.

In a general case, the differential equation governing the equation of state can be derived from the Friedmann equations,

$\mathrm{d} \ln \rho_{\mathrm{q}}=\frac{3(1+w)}{1+z} \mathrm{~d} z$

Then,

$\rho_{\mathrm{q}}=\rho_{\mathrm{q} 0} \exp 3 \int_{0}^{z} \frac{1+w}{1+z} \mathrm{~d} z \equiv \rho_{\mathrm{q} 0} \exp Q(z)$

where $Q(z)$ depends on $w(z)$.

With the hypotheses of null curvature and only two equations of state, one of them for ordinary matter, (3) can be written as

$t=\frac{1}{H_{0}} \int_{z}^{\infty} \frac{\mathrm{d} z}{(1+z) \sqrt{\Omega_{\mathrm{m} 0}(1+z)^{3}+\Omega_{\mathrm{q} 0} \exp Q}}$,

where $\Omega_{\mathrm{q} 0}$ is the current adimensional dark energy density. The general redshift dependence of the adimensional dark energy density is then $\Omega_{\mathrm{q}}=\Omega_{\mathrm{q} 0} \exp Q$. From (6), the age, $t_{0}$, can be derived by setting the lower integral limit to $z=0$.

\subsection{A simple parameterization}

Given the absence of widely accepted and physically indisputable dark energy models, a polynomial parameterization has 
been used by some authors as a simpler and pragmatic way to approximate the time dependence of the equation of state. The dependence so parameterized could then be fitted to different model predictions.

For example, Goliath et al. (2001) and Linder (2001) considered linear models of the form

$w(z)=w_{0}+w_{1} z$,

so that

$\exp Q=(1+z)^{3\left(1+w_{0}-w_{1}\right)} \exp 3 w_{1} z$.

Linder (2003) also proposed the form

$w(z)=w_{0}+\frac{w_{a} z}{1+z}$

which converges to $w_{0}+w_{a}$ at high redshift and gives

$\exp Q=(1+z)^{3\left(1+w_{0}+w_{a}\right)} \exp \frac{-3 w_{a} z}{1+z}$.

Both forms were introduced on the basis of reducing the number of parameters to two. Assuming that the above expressions can be extrapolated to $z \rightarrow \infty$, an assumption otherwise needed to evaluate the age of the universes that they generate, they can be used to reinforce the need either to assume phantom energies as dark energies or to revise the basic cosmological parameters $H_{0}, \Omega_{\mathrm{m} 0}$ or $t_{\mathrm{GC}}, t_{\mathrm{f}}$.

Although the age of the Universe cannot be used on its own to constrain two parameters, additional constraints may be invoked.

A strong constrain is that dark energies cannot dominate at high redshift. Otherwise, the expansion would be different and primordial nucleosynthesis would yield different helium abundances and the period of structure formation would be affected as well (Doran et al. 2001). This constraint may be formally represented by $\rho_{\mathrm{q}} / \rho_{\mathrm{m}} \rightarrow 0$ for $z \rightarrow \infty$, which gives, using Eqs. (5), (8) and (10), the conditions $w_{1}<0$ and $w_{0}+w_{a}<0$ for Eqs. (7) and (9), respectively. This strong constraint implies that for an equation of state of the form (7) phantom energies will necessarily be present at high redshift.

A weaker constraint, since it is not based on observational evidence, is that an event horizon should not be present since it poses problems for string theories. This is equivalent to a future non-accelerated universe. Formally, $\rho_{\mathrm{q}} /(1+z)^{2} \rightarrow$ constant for $z \rightarrow-1$. This condition gives, using Eqs. (5), (8) and (10), the conditions $1+3\left(w_{0}-w_{1}\right)>0$ and $w_{a}>0$ for Eqs. (7) and (9), respectively.

Figures 2 and 3 show the pairs of values for $w_{0}$ and $w_{1}$ or $w_{a}$, for equations of state of the form (7) or (9), respectively, that give an age of the Universe compatible with the minimum values derived from globular clusters at $95 \%$ and $68 \%$ c.l., assuming that globular clusters were formed at $z=3$ in a flat universe with $\Omega_{\mathrm{m} 0}=0.33$. The regions where dark energy densities grow unacceptably high at high redshifts are marked. In both cases long epochs where $w<-1$ are present, except for some $w_{0}, w_{a}$ pairs corresponding to the lowest $98 \%$ c.l. age values (Fig. 3). Nominal age values produce even more negative $w$ values. Hence, the conclusions of the previous section that favoring a cosmological constant as dark energy would probably require reducing $t_{\mathrm{GC}}, H_{0}$ or both, and possibly advancing the epoch of globular cluster formation, are reinforced.

\section{Age-redshift relations}

The results of Table 1 show that the upper $w$ limits range between -0.23 and -1.21 . The first limit does not accelerate the Universe, since for this effect to happen $w<-1 / 3$ is required. Hence the most extreme upper limit for an accelerated universe may be taken as $w=-1 / 3$. The other extreme may be approximated by $w=-4 / 3$, since values of $w=n / 3$, where $n$ is an integer, make obtaining analytical solutions easier. Four nullcurvature cases will therefore be considered: the quintessence limit $w=-1 / 3$, the quintessence $w=-2 / 3$, the cosmological constant $w=-1$ and the phantom energy $w=-4 / 3$. These should be considered as weighted mean approximations to $w$.

\subsection{Quintessence limit ( $w=-1 / 3)$}

As can be deduced from Friedmann equations, this equation of state corresponds to a universe with $\ddot{a}=0$. This is a nonaccelerated universe corresponding to the limit between the decelerated and accelerated cases. Integrating (3),

$$
\begin{aligned}
t= & \frac{1}{H_{0}}\left[\frac{\sqrt{\Omega_{\mathrm{m} 0}(1+z)+\Omega_{\mathrm{w} 0}}}{\Omega_{\mathrm{w} 0}(1+z)}\right. \\
& \left.+\frac{\Omega_{\mathrm{m} 0}}{\Omega_{\mathrm{w} 0}^{3 / 2}} \ln \left(\sqrt{1+\frac{\Omega_{\mathrm{w} 0}}{\Omega_{\mathrm{m} 0}(1+z)}}-\sqrt{\frac{\Omega_{\mathrm{w} 0}}{\Omega_{\mathrm{m} 0}(1+z)}}\right)\right],
\end{aligned}
$$

from which the age of the Universe can be derived:

$t_{0}=\frac{1}{H_{0}}\left[\frac{1}{\Omega_{\mathrm{w} 0}}+\frac{\Omega_{\mathrm{m} 0}}{\Omega_{\mathrm{w} 0}^{3 / 2}} \ln \left(\frac{1-\sqrt{\Omega_{\mathrm{w} 0}}}{\sqrt{\Omega_{\mathrm{m} 0}}}\right)\right]$.

\subsection{Quintessence $(w=-2 / 3)$}

The integration of (3) give (Grössner \& Hofreiter 1975):

$$
\begin{aligned}
t=\frac{1}{H_{0}} & \left\{\frac{2 \sqrt{\Omega_{\mathrm{m} 0}(1+z)^{2}+\Omega_{\mathrm{w} 0}}}{\Omega_{\mathrm{w} 0}(1+z)+\sqrt{\Omega_{\mathrm{m} 0} \Omega_{\mathrm{w} 0}(1+z)^{3}}}\right. \\
& \left.+2 \varepsilon \sqrt[4]{\frac{\Omega_{\mathrm{m} 0}}{\Omega_{\mathrm{w} 0}^{3}}}[F(\phi, k) / 2-E(\phi, k)]\right\},
\end{aligned}
$$

where $F(\phi, k)$ and $E(\phi, k)$ are elliptic integrals of the first and second kinds, respectively, of modulus $k=\sin \pi / 4$ and amplitude $\phi$ given by

$\sin \phi=\frac{2 \sqrt[4]{\Omega_{\mathrm{w} 0} \Omega_{\mathrm{m} 0}} \sqrt{1+z}}{\sqrt{\Omega_{\mathrm{w} 0}}+\sqrt{\Omega_{\mathrm{m} 0}}(1+z)}$

for $-\pi / 2 \leq \phi \leq \pi / 2$, with $\varepsilon$ a sign factor

$\varepsilon=\left\{\begin{array}{l}+1 \text { for } 0 \leq \sqrt{\frac{\Omega_{\mathrm{w} 0}}{\Omega_{\mathrm{m} 0}}} \leq 1+z \\ -1 \text { for } 1+z \leq \sqrt{\frac{\Omega_{\mathrm{w} 0}}{\Omega_{\mathrm{m} 0}}} \leq \infty .\end{array}\right.$

The value $1+z=\left(\Omega_{\mathrm{w} 0} / \Omega_{\mathrm{m} 0}\right)^{1 / 2}$ corresponds to the coasting point where $\ddot{a}=0$, which coincides with the redshift at which the matter and the quintessence energy densities are equal.

Equation (13) with $\varepsilon=+1$ is then valid for epochs before the coasting point. After the coasting point (as the 




Fig. 2. Possible pairs $\left(w_{0}, w_{1}\right)$ defining a dark energy equation of state $w(z)=w_{0}+w_{1} z$ that give an age of 12 and 13.4 Gyr corresponding to the minimum age of the Universe at $95 \%$ and $68 \%$ confidence levels, respectively. A null-curvature universe with ordinary matter, $\Omega_{\mathrm{m} 0}=0.33$ and $t_{\mathrm{f}}=2 \mathrm{Gyr}$ has been assumed. The regions $w_{1}>0$ and $1+3\left(w_{0}-w_{1}\right)<0$ define respectively the parameter space were dark energy becomes unacceptably high at high redshifts and an event horizon exists.

present epoch would be were our universe dominated by this quintessence), it is straightforward to demonstrate that to the value of (13) for $\varepsilon=-1$ twice the value of the second term of (13) for $\varepsilon=+1$ has to be added. For example, for $z=0$ it is possible to obtain the age of this universe,

$$
\begin{aligned}
t_{0}= & \frac{1}{H_{0}}\left\{\frac{2}{\Omega_{\mathrm{w} 0}+\sqrt{\Omega_{\mathrm{m} 0} \Omega_{\mathrm{w} 0}}}+2 \sqrt[4]{\frac{\Omega_{\mathrm{m} 0}}{\Omega_{\mathrm{w} 0}^{3}}}\right. \\
& \times[K(k)-2 E(k)-F(\phi, k) / 2+E(\phi, k)]\},
\end{aligned}
$$

where $K(k)$ and $E(k)$ are the complete elliptic functions of first and second kinds, respectively, and

$$
\sin \phi=\frac{2 \sqrt[4]{\Omega_{\mathrm{w} 0} \Omega_{\mathrm{m} 0}}}{\sqrt{\Omega_{\mathrm{w} 0}}+\sqrt{\Omega_{\mathrm{m} 0}}}
$$

\subsection{The cosmological constant}

The integration of (3) gives

$t=\frac{1}{3 H_{0} \sqrt{\Omega_{\Lambda 0}}} \ln \left(\frac{\sqrt{\Omega_{\mathrm{m} 0}(1+z)^{3}+\Omega_{\Lambda 0}}+\sqrt{\Omega_{\Lambda 0}}}{\sqrt{\Omega_{\mathrm{m} 0}(1+z)^{3}+\Omega_{\Lambda 0}}-\sqrt{\Omega_{\Lambda 0}}}\right)$.
This provides the known equation for the age of the Universe by taking $z=0$,

$t_{0}=\frac{2}{3 H_{0} \sqrt{\Omega_{\Lambda 0}}} \ln \left(\frac{1+\sqrt{\Omega_{\Lambda 0}}}{\sqrt{\Omega_{\mathrm{m} 0}}}\right)$.

It is interesting to note that, for $\Omega_{\mathrm{m} 0}=0.2628$ and $\Omega_{\Lambda 0}=$ 0.7372 ,

$t_{0}=\frac{1}{H_{0}}$

This generates a curious "cosmic coincidence" since these densities are within the range currently assumed as the solution for our Universe, in particular very close to those assumed by Spergel et al. (2003). Equation (19) could then be used as a good approximation for the age of the Universe, were it dominated by a cosmological constant.

Finally, for this equation of state, (17) can be rewritten to give the evolution of the scale factor

$a=a_{0} \sqrt[3]{\frac{\Omega_{\mathrm{m} 0}}{\Omega_{\Lambda 0}} \sinh ^{2}\left(\frac{3 H_{0} \sqrt{\Omega_{\Lambda 0}} t}{2}\right)}$. 


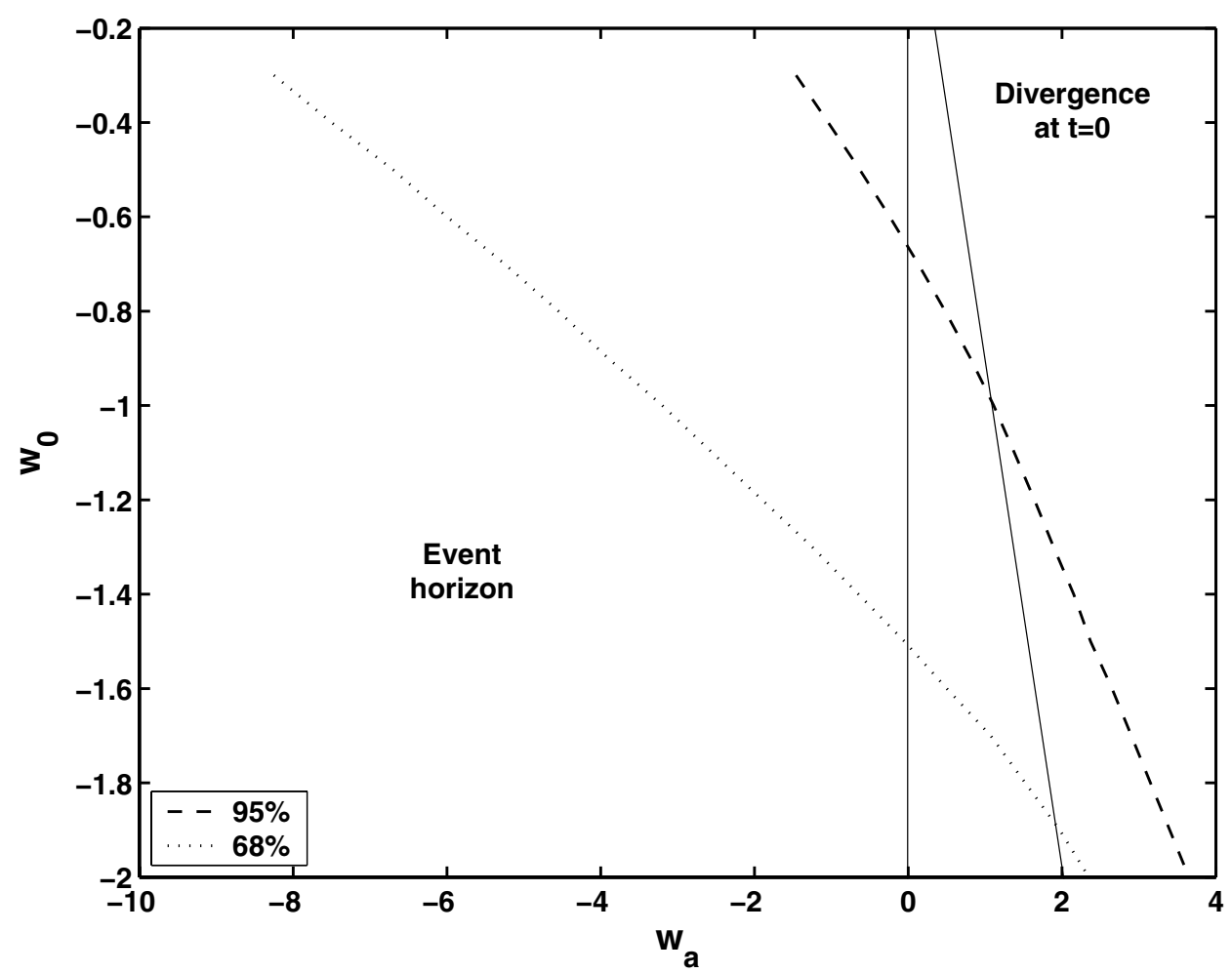

Fig. 3. Possible pairs $\left(w_{0}, w_{a}\right)$ defining a dark energy equation of state $w(z)=w_{0}+w_{a} z /(1+z)$ that give an age of 12 and 13.4 Gyr corresponding to the minimum age of the Universe at $95 \%$ and $68 \%$ confidence levels, respectively. A null-curvature universe with ordinary matter, $\Omega_{\mathrm{m} 0}=0.33$ and $t_{\mathrm{f}}=2 \mathrm{Gyr}$ has been assumed. The regions $w_{0}+w_{a}>0$ and $w_{a}<0$ define respectively the parameter space were dark energy becomes unacceptably high at high redshifts and an event horizon exists.

\subsection{Phantom energy $(w=-4 / 3)$}

From the integration of (3) it is possible to obtain (Grössner \& Hofreiter 1975)

$$
\begin{aligned}
t= & \frac{\sqrt{2-\sqrt{2}}}{2 H_{0} \sqrt[8]{\Omega_{\mathrm{m} 0} \Omega_{\mathrm{w} 0}^{3}}} \\
& \times\left\{\frac{\sqrt{2}}{2}\left[F\left(\phi_{1}, k\right)-K(k)\right]-\varepsilon\left[F\left(\phi_{2}, k\right)-K(k)\right]\right\},
\end{aligned}
$$

where the modulus $k=\sqrt{2}-1$ and the amplitudes are

$$
\sin \phi_{1}=\frac{\left(\frac{\Omega_{\mathrm{w} 0}}{\Omega_{\mathrm{m} 0}}\right)+(1+z)^{4}-2 \sqrt{2} \sqrt[4]{\frac{\Omega_{\mathrm{w} 0}}{\Omega_{\mathrm{m} 0}}}(1+z)\left(\sqrt[4]{\frac{\Omega_{\mathrm{w} 0}}{\Omega_{\mathrm{m} 0}}}+1+z\right)^{2}}{\left(\frac{\Omega_{\mathrm{w} 0}}{\Omega_{\mathrm{m} 0}}\right)+(1+z)^{4}+2 \sqrt{2} \sqrt[4]{\frac{\Omega_{\mathrm{w} 0}}{\Omega_{\mathrm{m} 0}}}(1+z)\left(\sqrt[4]{\frac{\Omega_{\mathrm{w} 0}}{\Omega_{\mathrm{m} 0}}}+1+z\right)^{2}}
$$

and

$\sin \phi_{2}=\frac{\left(\sqrt[4]{\frac{\Omega_{\mathrm{w} 0}}{\Omega_{\mathrm{m} 0}}}-(1+z)\right)^{2}-\sqrt{2} \sqrt[4]{\frac{\Omega_{\mathrm{w} 0}}{\Omega_{\mathrm{m} 0}}}(1+z)}{\left(\sqrt[4]{\frac{\Omega_{\mathrm{w} 0}}{\Omega_{\mathrm{m} 0}}}-(1+z)\right)^{2}+\sqrt{2} \sqrt[4]{\frac{\Omega_{\mathrm{w} 0}}{\Omega_{\mathrm{m} 0}}}(1+z)}$,

with $-\pi / 2 \leq \phi_{1}, \phi_{2} \leq \pi / 2$ and $\varepsilon$, where

$\varepsilon=\left\{\begin{array}{l}+1 \text { for } 0 \leq \sqrt[4]{\frac{\Omega_{\mathrm{w} 0}}{\Omega_{\mathrm{m} 0}}} \leq 1+z \\ -1 \text { for } 1+z \leq \sqrt[4]{\frac{\Omega_{\mathrm{w} 0}}{\Omega_{\mathrm{m} 0}}} \leq \infty .\end{array}\right.$
The same comments as for the case $w=-2 / 3$ apply for the meaning of $\varepsilon$. The age of the Universe is then

$$
t_{0}=\frac{\sqrt{2-\sqrt{2}}}{2 H_{0} \sqrt[8]{\Omega_{\mathrm{m} 0} \Omega_{\mathrm{w} 0}^{3}}}\left\{\frac{\sqrt{2}}{2} F\left(\phi_{1}, k\right)+F\left(\phi_{2}, k\right)+K(k)\left(3-\frac{\sqrt{2}}{2}\right)\right\},
$$

with the values of $\phi_{1}$ and $\phi_{2}$ given by Eqs. (22) and (23) for $z=0$.

The value $1+z=\left(\Omega_{\mathrm{w} 0} / \Omega_{\mathrm{m} 0}\right)^{1 / 4}$ corresponds to the redshift at which the energy densities of dark and conventional matter are equal. The coasting point where $\ddot{a}=0$ is given by $1+z=$ $\left(3 \Omega_{\mathrm{w} 0} / \Omega_{\mathrm{m} 0}\right)^{1 / 4}$.

Again, Eq. (19) provides a good estimate for the age of the Universe when $w=-4 / 3$ for $\Omega_{\mathrm{m} 0}=0.3054$ and $\Omega_{\mathrm{w} 0}=0.6946$.

Figure 4 gives the age-redshift relation for each equation of state for $\Omega_{\mathrm{m} 0}=0.33$ and $\Omega_{\mathrm{w} 0}=0.67$. Figure 5 gives the age of the Universe for each equation of state versus $\Omega_{\mathrm{m} 0}$. Table 2 gives the age of the Universe derived for each equation of state considered. Abramowitz \& Stegun (1972) has been used for the evaluation of elliptical integrals.

\section{Summary}

In the most accepted cosmological model the Universe is currently accelerated owing to a form of dark energy of unknown 




Fig. 4. Age-redshift relations in null-curvature universes with ordinary matter for each of the dark energy equations of state $w$ considered and $\Omega_{\mathrm{m} 0}=0.33$.

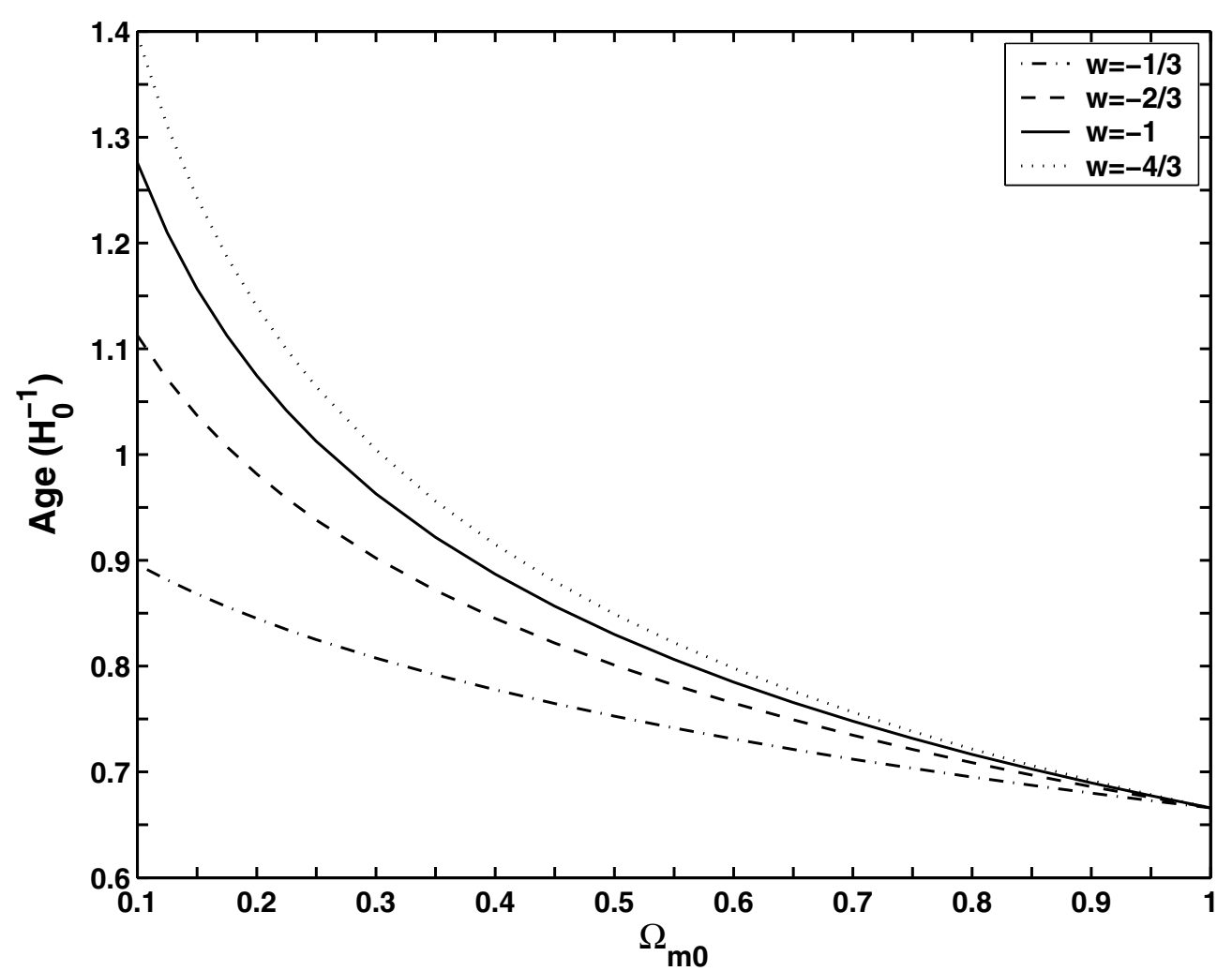

Fig. 5. Age of null-curvature universes with ordinary matter versus $\Omega_{\mathrm{m} 0}$ for each of the dark energy equations of state $w$ considered. 
Table 2. Values of $H_{0} t_{0}$ for the different equations of state of dark energy and different $\Omega_{\mathrm{m} 0}$ for $\epsilon=0$.

\begin{tabular}{ccccc}
\hline \hline$w$ & $\Omega_{\mathrm{m} 0}=0.24$ & $\Omega_{\mathrm{m} 0}=0.27$ & $\Omega_{\mathrm{m} 0}=0.30$ & $\Omega_{\mathrm{m} 0}=0.33$ \\
\hline$-1 / 3$ & 0.8302 & 0.8191 & 0.8088 & 0.7991 \\
$-2 / 3$ & 0.9473 & 0.9241 & 0.9033 & 0.8844 \\
-1 & 1.0251 & 0.9927 & 0.9641 & 0.9386 \\
$-4 / 3$ & 1.0790 & 1.0399 & 1.0057 & 0.9754 \\
\hline
\end{tabular}

origin, as found via distance-redshift fits to high redshift SNeIa. This energy complements the fraction of ordinary matter so that the curvature is null, according to the observed CMBR anisotropy and the inflation paradigm. Also, the dark energy increases the age of the Universe for the same values of $\Omega_{\mathrm{m} 0}$ and $H_{0}$ with respect to a universe composed of non-relativistic matter only, thus alleviating possible age conflicts.

In this article a recent determination of $\Omega_{\mathrm{m} 0}, t_{0}$ and $H_{0}$ has been selected from the literature with the criterion of providing a set of cosmological parameters as model-independent as possible. A simple analysis shows that the weighted mean equation of state of dark energy has upper values in the range $-0.23>w>-1.21$. Hence, not only a cosmological constant but also quintessence or phantom energy are also viable dark energy candidates from an observational point of view. In fact, the mean values of $\Omega_{\mathrm{m} 0}, t_{0}$ and $H_{0}$ favor phantom energy over other alternatives. Moreover, a time-dependent $w$ favors even more the existence of epochs with $w<-1$. It is qualitatively shown that to reduce the likelihood of phantom energy to favor a cosmological constant as dark energy, smaller globular cluster ages and/or smaller $H_{0}$ values with respect to those currently determined are required. Pushing back globular cluster formation to earlier epochs cannot be ruled out, but only together with lower values of $t_{\mathrm{GC}}$ and/or $H_{0}$. Otherwise, an age conflict might still be present.

The range of upper $w$ limits inferred is approximately covered by taking $w=-n / 3$ for $n=1,2,3,4$. For each of these constant values of $w$, which include quintessence, the cosmological constant and phantom energy, analytical age-redshift solutions for Euclidean universes have been deduced and analytical age expressions derived. Some of these analytical solutions have not been derived before. Furthermore, no analytical expressions seem to be available in the literature for the range of the constant equations of state considered. Analytical equations are more precise, faster and readier to use than numerical calculations when evaluating the age of the Universe. Moreover, the explicit dependence on $\Omega_{\mathrm{m} 0}$ and $\Omega_{\mathrm{w} 0}$ renders their study easier. Finally, curious "cosmic coincidences" make $1 / H_{0}$ a good approximation for the age of the Universe for the currently accepted $\Omega_{\mathrm{m} 0}$ values assuming null curvature and a cosmological constant or a phantom energy as dark energies.

\section{References}

Abramowitz, M., \& Stegun, I. A. 1972, Handbook of Mathematical Functions (Dover Publications, Inc.)

Aurich, R., \& Steiner, F. 2003, Phys. Rev. D, 67, 123511

Bolte, M., \& Hogan, C. J. 1995, Nature, 376, 399

Caldwell, R. R. 1999 [arXiv: astro-ph/9908168]

Caldwell, R. R., Dave, R., \& Steinhardt, P. J. 1998, Phys. Rev. Lett., 80,1582

Carlberg, R. G., Yee, H. K. C., Ellingson, E., et al. 1996, ApJ, 462, 32

Carretta, E., Gratton, R. G., Clementini, G., \& Fusi Pecci, F. 2000, ApJ, 533, 215

Carroll, S. M., Hoffman, M., \& Trodden, M. 2003, Phys. Rev. D, 68, 023509

Cline, J. M. 2001, JHEP, 8, 35

de Bernardis, P., Ade, P. A. R., \& Bock, J. J. 2000, Nature, 404, 955

del Popolo, A. 2003, A\&A, 408, 43

Di Pietro, E., \& Claeskens J.-F. 2003, MNRAS, 341, 1299

Doran, M., Lilley, M., Schwindt, J., \& Wetterich, C. 2001, ApJ, 559, 501

Doran, M., \& Lilley, M. 2002, MNRAS, 330, 965

Fassnacht, C. D., Xanthopoulos, E., Koopmans, L. V. E., \& Rusin, D. 2002, ApJ, 581, 823

Freedman, W., Madore, B. F., Gibson, B. K., et al. 2001, ApJ, 553, 47

Goliath, A., Amanulah, R., Astier, P., Goobar, A., \& Pain, R. 2001, A\&A, 380, 6

González-Díaz, P. F. 2003, Phys. Rev. D, 68, 021303

Grössner, W., \& Hofreiter, N. 1975, Integraltafel. Erster Teil. Unbestimmte Integrale (Springer-Verlag)

Hawking, S. W., \& Ellis, G. F. R. 1973, The large scale structure of Space Time (Cambridge University Press)

Hogg, D. W. 2000 [arXiv: astro-ph/9905116]

Hu, W., Fukugita, M., Zaldarriaga, M., \& Tegmark, M. 2001, ApJ, 549,669

Kneller, J. P., \& Strigari, L. E. 2003, Phys. Rev. D, 68, 083517

Knox, L., Christensen, N., \& Skordis, C. 2001, ApJ, 563, L95

Krauss, L. M., \& Chaboyer, B. 2003, Science, 299, 65

Linder, E. V. 2001 [arXiv: astro-ph/0108280]

Linder, E. V. 2003, Phys. Rev. Lett., 90, 091301

Mauskopf, P. D., Ade, P. A. R., Allen, S. W., et al. 2000, ApJ, 538, 505

Parodi, B. R., Saha, A., Sandage, A., \& Tammann, G. A. 2000, ApJ, 540, 634

Perlmutter, S., Turner, M. S., \& White, M. 1999, Phys. Rev. Lett., 83, 670

Ratra, B., Peebles, P. J. E. 1998, Phys. Rev. D, 37, 3406

Reichart, D. E., Castander, F. J., \& Nichol, R. C. 1999, ApJ, 516, 1

Riess, A. G., Filippenko, A. V., Challis, P., et al. 1998, AJ, 116, 1009

Riess, A. G., Nugent, P. E., Gilliland, R. L., et al. 2001, ApJ, 560, 49

Spergel, D. N., Verde, L., Peiris, H. V., et al. 2003, ApJS, 148, 175

Thomas, R. C., \& Kantowski, R. 2000, Phys. Rev. D, 62, J3507

Turner, M. S. 2002, ApJ, 576, L101

van den Bergh, S. 2001, ApJ, 559, L113

Waga, I., \& Miceli, A. P. M. R. 1999, Phys. Rev. D, 59, J3507

Wetterich, C. 1998, Nucl. Phys. B, 302, 668

Acknowledgements. This work has been supported by the Spanish Plan Nacional de Astronomía y Astrofísica under grant AYA200201379. This research has made use of NASA's Astrophysics Data System. 